\title{
Astragaloside IV attenuates penicillin-induced epilepsy via inhibiting activation of the MAPK signaling pathway
}

\author{
XINGMING ZHU, YANG CHEN, YUPING DU, QI WAN, YAN XU and JIAYU WU \\ Department of Neurology, 101st Hospital of Chinese People's Liberation Army, Wuxi, Jiangsu 214044, P.R. China
}

Received March 23, 2016; Accepted February 2, 2017

DOI: $10.3892 / \mathrm{mmr} .2017 .7896$

\begin{abstract}
Astrocytes perform several functions in the brain and spinal cord. Penicillin is commonly used for establishment of experimental epilepsy models. Previous studies have demonstrated that astragaloside IV (3-o- $\beta$-dxylopyranosyl-6-o- $\beta$-d-glucopyranosyl-cycloastragenol;

AS-IV) has comprehensive pharmacological functions on the attenuation of inflammation. In the present study, primary astrocyte cell cultures were divided into three groups: Control group, penicillin $(2,500 \mu \mathrm{M})$ treatment group (epilepsy model), and penicillin+AS-IV (20, 40, 80 and $160 \mu \mathrm{mol} / \mathrm{l})$ treatment group. The expression levels of inflammatory factors, including interleukin-1 $\beta$ and tumor necrosis factor- $\alpha$, were determined in the groups using western blot and reverse transcription-quantitative polymerase chain reaction analyses. The levels of members of the phosphorylated-mitogen-activated protein kinase (p-MAPK) family, including $\mathrm{p}$-c-Jun $\mathrm{N}$-terminal kinase $1 / 2$, p-extracellular signal-regulated protein kinase $1 / 2$ and p-p38, were determined using western blot analysis. Cell viability of the astrocytes was detected using a 3-(4,5-dimethyl-2-thiazolyl)-2,5-diphenyl-2-H-tetrazolium bromide assay and cell proliferation was evaluated using a Cell Counting Kit-8 assay. The results revealed that AS-IV significantly suppressed the expression of penicillin-induced inflammatory factors in the astrocytes at the transcriptional and translational levels, and occurred in a dose-dependent manner. The penicillin-induced increase in the protein levels of the the p-MAPK family were notably decreased by AS-IV. In addition, the penicillin-induced downregulation of primary astrocyte viability/cell proliferation was
\end{abstract}

Correspondence to: Dr Xingming Zhu or Dr Yuping Du, Department of Neurology, 101st Hospital of Chinese People's Liberation Army, 101 Xingyuanbei Road, Wuxi, Jiangsu 214044, P.R. China

E-mail: zhuxingmingwx@sina.com

E-mail: 813796909@qq.com

Key words: penicillin-induced epilepsy, astragaloside IV, inflammatory cytokine, mitogen-activated protein kinase family, astrocyte significantly reversed by the administration of AS-IV. From these results, it was concluded that AS-IV suppressed the penicillin-induced upregulation of inflammatory factors and p-MAPK in astrocytes, ultimately attenuating epilepsy.

\section{Introduction}

As a group of neurological diseases, epilepsy is characterized by epileptic seizures $(1,2)$. Worldwide, $1 \%(65,000,000$ individuals) of the population suffer with epilepsy (3), with almost $80 \%$ of new cases occurring in developing countries (4). In 2013 , the mortality rate was $\sim 116,000$, which was an increase from 112,000 in 1990 (5).

Astrocytes initiate, regulate and amplify immune-mediated mechanisms, and are associated with several diseases of the human central nervous system, including epilepsy $(6,7)$. In vitro studies have documented the ability of astrocytes, particularly active astrocytes, to produce cytokines, including interleukin (IL)-1 $\beta$ and tumor necrosis factor (TNF)- $\alpha$, which are expressed at high levels in experimental and human epileptogenic brain tissues $(8,9)$.

As a common antibiotic, penicillin functions as a chemical convulsant for the establishment of an experimental epilepsy model $(10,11)$. At least $100-5,000 \mu \mathrm{M}$ penicillin is required to inhibit GABA (12).

It is understood that astragaloside IV (3-o- $\beta$-d-xylopy ranosyl-6-o- $\beta$-d-glucopyranosyl-cycloastragenol; AS-IV), the primary pure saponin isolated from the root of Astragalus membranaceus, is an effective compound with distinct pharmacological effects, including anti-inflammatory effects $(13,14)$. To the best of our knowledge, the protective effects of AS-IV on epilepsy remain to be elucidated, therefore, the present study aimed to investigate the effect of AS-IV in a primary astrocyte model of penicillin-induced epilepsy.

\section{Materials and methods}

Cell culture. Primary astrocytes were derived from 1-5 day postnatal Sprague-Dawley male rats $(n=10)$. Neonatal rats were purchased from the Model Animal Research Center of Nanjing University. Briefly, neonatal rats were anesthetized and sacrificed by alcohol immersion. Following removal of the meninges and blood vessels, the cerebral cortices were collected and minced in $20 \mu \mathrm{g} / \mathrm{ml}$ DNase and $0.3 \%$ bovine 
serum albumin (BSA)-containing medium (Sigma-Aldrich; Merck KGaA, Darmstadt, Germany). The animal protocol was approved by Wuxi Laboratory Animal Management and the Animal Ethics Committee. All animal experiments were carried out in accordance with the ethical guidelines of the Ethics Committee of Jiangnan University (Wuxi, China).

The tissues were digested in $0.25 \%$ trypsin/EDTA solution for $30 \mathrm{~min}$ at $37^{\circ} \mathrm{C}$. The tissues were centrifuged ( $300 \mathrm{x} \mathrm{g}, 5 \mathrm{~min}$ at room temperature) and digested in $0.25 \%$ trypsin/EDTA solution for $30 \mathrm{~min}$ at $37^{\circ} \mathrm{C}$. The suspension was filtered through a $70 \mu \mathrm{m}$ nylon filter, pelleted by centrifugation ( $600 \mathrm{x} \mathrm{g}, 5 \mathrm{~min}$ at room temperature) to remove trypsin, and then suspended in $10 \%(\mathrm{v} / \mathrm{v})$ fetal bovine serum (FBS; Gibco; Thermo Fisher Scientific, Inc., Waltham, MA, USA) in Dulbecco's modified Eagle's medium/F12 (DMEM/F12; Gibco; Thermo Fisher Scientific, Inc.) containing a penicillin and streptomycin antibiotic mixture. The mixture was transferred to flasks and incubated under conditions of $37^{\circ} \mathrm{C}, 5 \% \mathrm{CO}_{2}$ and $90 \%$ relative humidity. When the cells reached confluence, the flasks were gently shaken to remove microglia and oligodendrocytes. Following shaking, the astrocytes were rinsed with phosphate-buffered saline (PBS) three times and trypsinized, followed by physical loosening of the astrocytes. The medium was removed and the astrocytes were placed in new flasks for culture in medium (DMEM/F12, 15\% FBS, L-glutamine and $500 \mathrm{ng} / \mathrm{ml}$ insulin) until they were confluent. The cells were trypsinized for subsequent experiments.

For primary astrocyte culture, $2,500 \mu \mathrm{M}$ (500 IU) of penicillin was used (15). Different concentrations of AST-IV $(20,40,80$ and $160 \mu \mathrm{mol} / \mathrm{l})$ were administrated $2 \mathrm{~h}$ prior to penicillin treatment. At $12 \mathrm{~h}$ following treatment with penicillin, the primary astrocytes were used to perform a series of experiments.

Western blot analysis. To determine temporal expression profiles of $\beta$-actin $(1: 1,000, \# 3700$, Cell Signaling Technology, Inc., Danvers, MA, USA), IL-1 $\beta$ (1:1,000, \#5204, Cell Signaling Technology, Inc.), TNF- $\alpha$ (1:1,000, \#11948, Cell Signaling Technology) and phosphorylated extracellular signal-regulated kinase (p-ERK, 1:1,000, \#8544, Cell Signaling Technology, Inc.)/c-Jun N-terminal kinase (p-JNK, 1:2,000, \#9255, Cell Signaling Technology, Inc.)/p-P38 (1:1,000, \#4511, Cell Signaling Technology, Inc.) mitogen-activated protein kinases (MAPKs), the astrocyte extract lysates were collected and analyzed. Briefly, the samples were washed with PBS rapidly and homogenized in RIPA lysis buffer containing a cocktail of protease inhibitors and phosphatase inhibitors (Roche Diagnostics, Nanjing, China). Protein concentration was determined and quantified using the bicinchoninic acid (Sigma-Aldrich) method. The sample lysates $(10 \mu \mathrm{g})$ were transferred and collected carefully for separation by $8 \%$ SDS-PAGE, following which they were electrotransferred onto polyvinylidene fluoride membranes (EMD Millipore, Bedford, MA, USA). The membranes were blocked in 5\% BSA for $1 \mathrm{~h}$ at room temperature, and incubated overnight at $4^{\circ} \mathrm{C}$ with primary antibodies, respectively. Following washing with TBST, the membranes were incubated with corresponding HRP-conjugated secondary antibodies (RPN4301, GE Healthcare Life Sciences, Little
Chalfont, UK) for $1 \mathrm{~h}$ at room temperature. $\beta$-actin was used as a loading control.

Reverse transcription-quantitative polymerase chain reaction ( $R T-q P C R)$ analysis. Total RNA was extracted by Trizol (Roche Diagnostics) method. RNA was reverse transcribed into cDNA using reverse transcriptase, in accordance with the manufacturer's protocol (Takara Biotechnology, Co., Ltd., Dalian, China). The sample used for RT-qPCR analysis comprised $2 \mu \mathrm{l}$ cDNA, $5 \mu \mathrm{l} 2 \mathrm{X}$ mix (Bio-Rad Laboratories, Inc., Hercules, CA, USA), $0.5 \mu 1$ forward primer, $0.5 \mu \mathrm{l}$ reverse primer and $2 \mu \mathrm{l}$ nanopure water to a final volume of $10 \mu \mathrm{l}$. The conditions for amplification were as follows: $10 \mathrm{sec}$ at $95^{\circ} \mathrm{C}$ for denaturation, $60 \mathrm{sec}$ at $60^{\circ} \mathrm{C}$ for annealing and extension, and 40 cycles for all primers. Experiments were performed in replicate. GAPDH was used as an endogenous control to normalize differences in the quantity of total RNA from each sample. The primer sequences were as follows: IL-1 $\beta$, forward 5'-TGAAATGCCACCTTTTGACAG-3' and reverse 5'-CCACAGCCACAATGAGTGATAC-3'; TNF- $\alpha$, forward 5'-CCTGTCTCTTCCTACCCAACC-3' and reverse 5'-GCAGGAGTGTCCGTGTCTTC-3'; GAPDH, forward 5'-CTTTGGTATCGTGGAAGGACTC-3' and reverse 5'-GTAGAGGCAGGGATGATGTTCT-3'. The comparative $\mathrm{Cq}\left(2^{-\Delta \triangle \mathrm{Cq}}\right)$ method was used to analyze the relative expression levels (16).

\section{3-(4,5-dimethyl-2-thiazolyl)-2,5-diphenyl-2-H-tetrazolium}

bromide (MTT) assay. Cell viability was measured using an MTT assay. The cells $(\sim 200 \mu \mathrm{l})$ at a concentration of $1 \times 10^{4} / \mathrm{ml}$ were seeded into 96 -well plates. Following incubation for $24 \mathrm{~h}$, $20 \mu \mathrm{l}$ of $5 \mathrm{mg} / \mathrm{ml}$ MTT solution was added to each well and the plate was incubated at $37^{\circ} \mathrm{C}$ for $4 \mathrm{~h}$. Subsequently, the medium was aspirated, the wells were washed with PBS and allowed to dry for $\sim 4 \mathrm{~h}$, following which $150 \mu \mathrm{l}$ DMSO was added to each well. The microtitre plate was placed on a shaker in order to dissolve the dye and absorbance was read at $450 \mathrm{~nm}$ using a Bio-Rad iMark plate reader (Bio-Rad Laboratories, Inc.).

Cell Counting Kit-8(CCK-8) assay. The numbers of cells were measured using a CCK-8 kit (Dojindo Molecular Technologies, Inc., Kumamoto, Japan). The cells $\left(\sim 5 \times 10^{3}\right)$ cells were seeded into 96-well plates. Into each well, $10 \mu 1$ CCK-8 solution was added and the cells were incubated at $37^{\circ} \mathrm{C}$ for $2 \mathrm{~h}$. The absorbance was read at $450 \mathrm{~nm}$ using a Bio-Rad iMark plate reader.

Statistical analysis. Differences among groups were analyzed using two-way analysis of variance followed by Bonferroni post hoc tests. Data were analyzed by SPSS software version 19.0 (IBM SPSS, Armonk, NY, USA) and presented as mean \pm standard error of the mean. $\mathrm{P}<0.05$ was considered to indicate a statistically significant difference.

\section{Results}

AS-IV significantly suppresses the translation of penicillin-induced inflammatory factors of primary astrocytes in a dose-dependent manner. Penicillin upregulated the protein levels of TNF- $\alpha$ and IL- $1 \beta$, whereas AS-IV (20, $40,80$ and $160 \mu \mathrm{mol} / \mathrm{l})$ dose-dependently suppressed the 
penicillin-induced upregulation in inflammatory factors (Fig. 1).

AS-IV significantly suppresses the transcription of penicillin-induced inflammatory factors of primary astrocytes in a dose-dependent manner. The changes in the transcription of inflammatory factors were consistent with those at the translational level. Only the higher doses of AS-IV (40, 80 and $160 \mu \mathrm{mol} / \mathrm{l})$ notably reduced the penicillin-induced upregulation of inflammatory factors (Fig. 2A and B).

Penicillin-induced protein levels of the p-MAPK family are decreased by AS-IV. AS-IV selectively reduced the penicillin-induced p-JNK and p-p38 MAPKs, but not p-ERK (Fig. 3).

Penicillin-induced downregulation of primary astrocyte viability is significantly increased following administration of AS-IV. The MTT assay revealed that, compared with the control group, the viability of the primary astrocytes in the penicillin-induced group was markedly downregulated. In addition, administration with the higher doses of AS-IV $(40,80$ and $160 \mu \mathrm{mol} / \mathrm{l})$ significantly increased cell viability (Fig. 4).

Penicillin-induced downregulation of primary astrocyte numbers is significantly elevated following administration of $A S-I V$. The results of the CCK8 assay revealed that, compared with the control group, the number of primary astrocytes in the penicillin-induced group was markedly reduced. The administration of higher doses of AS-IV (40, 80 and $160 \mu \mathrm{mol} / \mathrm{l})$ significantly reversed the penicillin-induced reduction in cell number (Fig. 5).

\section{Discussion}

As one of the most common serious neurological disorders (17), epilepsy is characterized by epileptic seizures $(1,2)$ and becomes more common as people age $(18,19)$. However, the exact mechanism underlying epilepsy remains to be elucidated (19). In normal conditions, brain electrical activity is modulated by various factors within and around neurons. Factors within neurons include the type, number and distribution of ion channels, and changes in receptor and gene expression (21). Factors around the neurons include ion concentrations, synaptic plasticity and the regulation of transmitter breakdown by glial cells $(21,22)$.

Astrocytes initiate, regulate and amplify immune-mediated mechanisms associated with epilepsy $(6,7)$. In vitro studies have confirmed the ability of active astrocytes to produce cytokines, including IL-1 $\beta$ and TNF- $\alpha$, which are expressed at high levels in experimental and human epileptogenic brain tissues $(8,9)$. Reactive astrogliosis was found to be a pathological hallmark of medically refractory epilepsy (23). Increasing evidence supports the hypothesis that activation of the innate immune response is involved in experimental and human epilepsy, and in the critical association of the inflammatory process in the etiopathogenesis of seizures $(8,24)$. Vezzani et al further examined the role of inflammation in epilepsy (25). The aim of the present study was to identify a
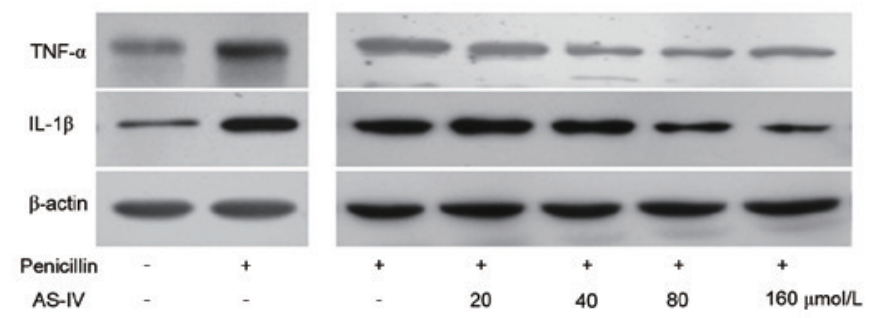

Figure 1. AS-IV dose-dependently suppresses penicillin-induced inflammatory factor upregulation at the protein level. Penicillin upregulated the protein levels of TNF- $\alpha$ and IL-1 $\beta$. AS-IV (20, 40, 80 and $160 \mu \mathrm{mol} / \mathrm{l})$ dose-dependently suppressed the penicillin-induced increase in inflammatory factors. AS-IV, astragaloside IV; TNF-tumor necrosis factor- $\alpha$; IL-1 $\beta$, interleukin-1 $1 \beta$.

novel method to attenuate epilepsy via inhibiting the inflammatory pathway.

AS-IV is an effective compound with distinct pharmacological anti-inflammatory effects $(13,14)$, however, the role of AS-IV in epilepsy remains to be fully elucidated. Penicillin has been demonstrated to function as a convulsant for the establishment of experimental epilepsy models $(10,11)$. The present study examined the effect of AS-IV against penicillin-induced epilepsy in primary astrocytes.

In the present study, penicillin was used to induce epilepsy in Primary astrocytes from SD rats, and the protein and mRNA levels of TNF- $\alpha$ and IL- $1 \beta$ were examined in different groups to investigate whether the model was successfully established. The results revealed that penicillin upregulated the protein and mRNA levels of TNF- $\alpha$ and IL-1 $\beta$, which were consistent with the results of previous studies showing the upregulation of pro-inflammatory cytokines $(25,26)$. The present study then examined whether AS-IV $(20,40,80$ and $160 \mu \mathrm{mol} / \mathrm{l})$ had effects on the levels of inflammatory factors using western blot and RT-qPCR analyses. The results revealed that AS-IV dose-dependently suppressed the penicillin-induced increase in inflammatory factors. These results suggested that the effects of AS-IV on ameliorating epilepsy were dependent on the reduced release of inflammatory factors from the cultured cells. These findings were consistent with previous studies. For example, it has been reported that transgenic mice with low-moderate overexpression of TNF- $\alpha$ in astrocytes exhibit reduced susceptibility to seizures (27), and mice lacking caspase-1, which is the biosynthetic enzyme of IL-1 $\beta$, are unable to release the biologically active form of IL- $1 \beta$ and also exhibit reduced seizure susceptibility (28). However, which proteins are involved in this process remain to be elucidated, and the present study performed further experiments to investigate this.

TNF- $\alpha$ is a cytokine released from activated astrocytes and microglia, and is closely associated with IL-1 $\beta$. In the hippocampus, IL-1 $\beta$ affects synaptic transmission, and inhibits long-term potentiation via the activation of JNK and p38 MAPK $(29,30)$. Therefore, the present study performed western blot analysis to examine the levels of p-MAPK in different groups. The results revealed that AS-IV decreased the penicillin-induced upregulation of p-p38 
A

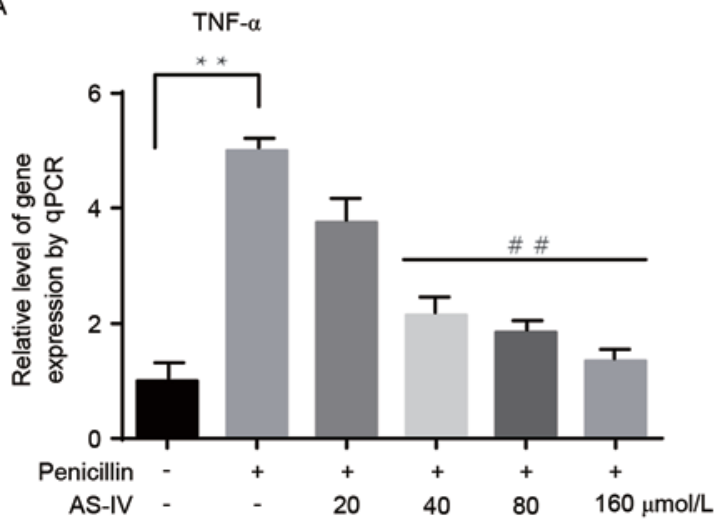

B

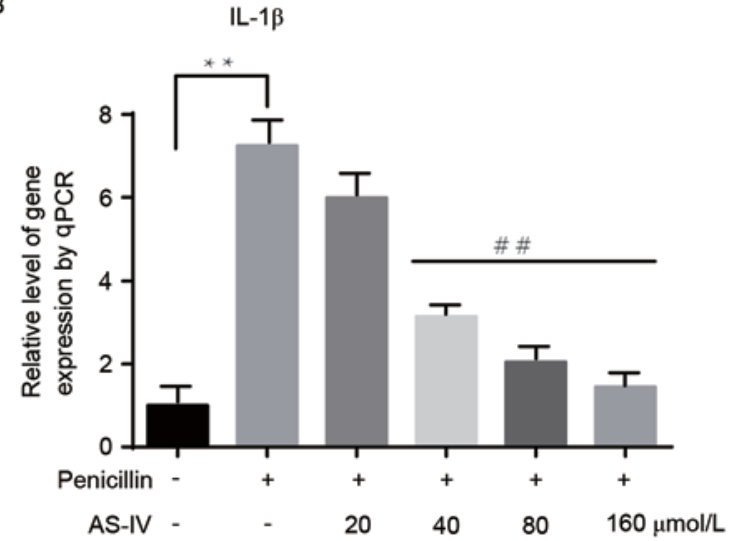

Figure 2. AS-IV dose-dependently suppresses penicillin-induced inflammatory factor elevation at the mRNA level. Penicillin upregulated the mRNA levels of (A) TNF- $\alpha$ and (B) IL-1 $\beta$. Only higher doses of AS-IV (40,80 and $160 \mu \mathrm{mol} / \mathrm{l})$ notably reduced the penicillin-induced upregulation in inflammatory factors (Fig. 2B). ${ }^{* *} \mathrm{P}<0.01$ penicillin group, vs. control group; ${ }^{* /} \mathrm{P}<0.01 \mathrm{AS}-\mathrm{IV}+$ penicillin group, vs. penicillin group. AS-IV, astragaloside IV; TNF-tumor necrosis factor- $\alpha$; IL-1 $\beta$, interleukin-1 $\beta$; qPCR, quantitative polymerase chain reaction.
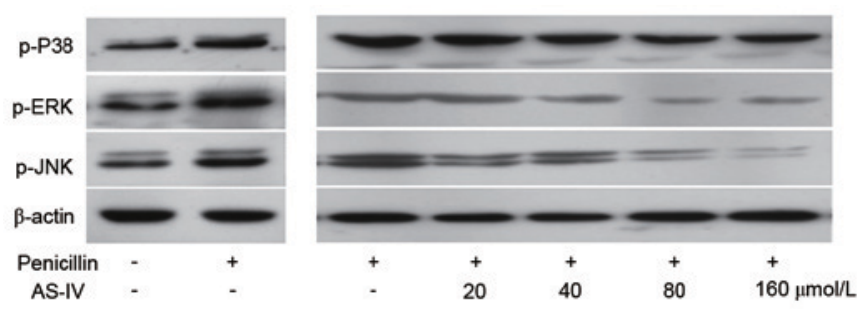

Figure 3. Penicillin-induced elevation in protein levels of the p-MAPK family are decreased by AS-IV. AS-IV selectively reduced the penicillin-induced upregulation of p-JNK and p-p38 MAPK, but not p-ERK. AS-IV, astragaloside IV; p-, phosphorylated; MAPK, mitogen-activated protein kinase; JNK, c-Jun N-terminal kinase; ERK, extracellular signal-regulated kinase.

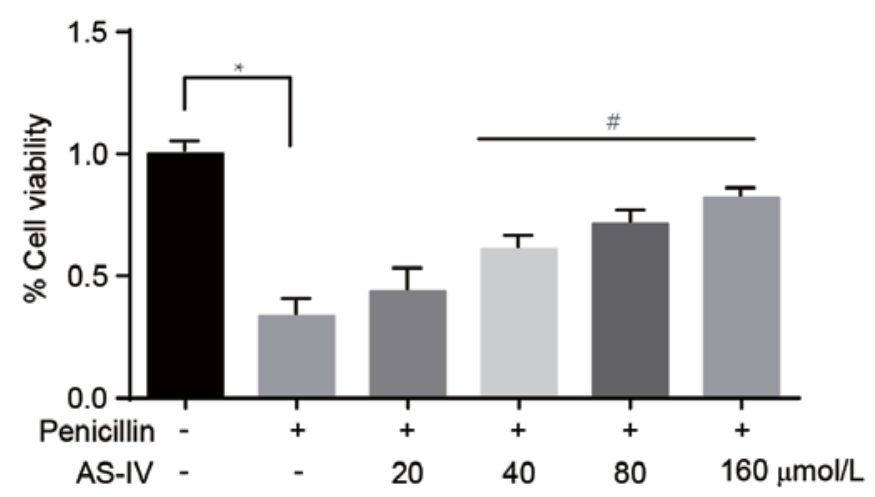

Figure 4. Penicillin-induced downregulation of primary astrocyte viability is significantly elevated by administration of AS-IV. Compared with the control group, cell viability of primary astrocytes in the penicillin group was downregulated. Higher doses of AS-IV (40, 80 and $160 \mu \mathrm{mol} / \mathrm{l})$ rescued cell viability. ${ }^{*} \mathrm{P}<0.01$ penicillin group, vs. control group; ${ }^{"} \mathrm{P}<0.01$ AS-IV+penicillin group, vs. penicillin group. AS-IV, astragaloside IV.

and $\mathrm{p}-\mathrm{JNK}$, which was in line with the results of previous studies $(29,30)$.

Taken together, the above results led to the conclusion that AS-IV suppressed penicillin-induced inflammatory factor

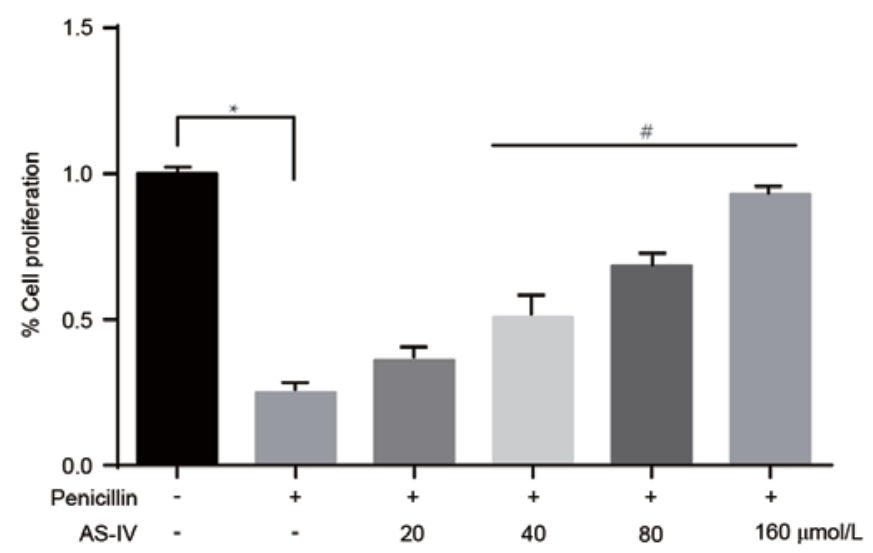

Figure 5. Penicillin-induced downregulation in primary astrocyte number is significantly elevated by administration of AS-IV. Compared with the control group, the number of primary astrocytes in the penicillin group was reduced. Higher doses of AS-IV (40, 80 and $160 \mu \mathrm{mol} / \mathrm{l})$ significantly reversed the penicillin-induced decrease in cell number. ${ }^{*} \mathrm{P}<0.01$ penicillin group, vs. control group; ${ }^{*} \mathrm{P}<0.01 \mathrm{AS}-\mathrm{IV}+$ penicillin group, vs. penicillin group. AS-IV, astragaloside IV.

release, and suppressed activation of the p-p38 and p-JNK MAPK signaling pathway.

It has been reported that cytokines have the ability to contribute to excitotoxic and apoptotic neuronal death (31), indicating the possibility of generating seizure-mediated neuronal damage. Therefore, the present study examined the effects of AS-IV on Primary astrocytes from SD rats. An MTT assay was performed, which revealed that the higher doses of AS-IV improved the viability of the primary astrocytes from SD rats. The effects of AS-IV on the proliferation of primary astrocytes were also determined using a CCK8 assay, the results of which were consistent with those of cell viability. Taken together, the results of the present study demonstrated that AS-IV suppressed the penicillin-induced release of inflammatory factors and suppressed activation of the MAPK signaling pathway, ultimately attenuating epilepsy. These findings provide a basis for further investigations of the therapeutic role of AS-IV in epilepsy via targeting astrocytes. 


\section{References}

1. Chang BS and Lowenstein DH: Epilepsy. N Engl J Med 349: 1257-1266, 2003.

2. Fisher RS, Acevedo C, Arzimanoglou A, Bogacz A, Cross JH, Elger CE, Engel J Jr, Forsgren L, French JA, Glynn M, et al: ILAE Official Report: A practical clinical definition of epilepsy. Epilepsia 55: 475-482, 2014.

3. Thurman DJ, Beghi E, Begley CE, Berg AT, Buchhalter JR, Ding D, Hesdorffer DC, Hauser WA, Kazis L, Kobau R, et al: Standards for epidemiologic studies and surveillance of epilepsy. Epilepsia 52 (Suppl 7): S2-S26, 2011.

4. Epilepsy. Fact Sheets. World Health Organization, 2012.

5. GBD 2013 Mortality and Causes of Death Collaborators: regional, and national age-sex specific all-cause and cause-specific mortality for 240 causes of death, 1990-2013: A systematic analysis for the Global Burden of Disease Study 2013. Lancet 385: 117-171, 2013.

6. Farina C, Aloisi F and Meinl E: Astrocytes are active players in cerebral innate immunity. Trends Immunol 28: 138-145, 2007.

7. Seifert G, Carmignoto G and Steinhäuser C: Astrocyte dysfunction in epilepsy. Brain Res Rev 63: 212-221, 2010.

8. Aronica E and Crino PB: Inflammation in epilepsy: Clinical observations. Epilepsia 52 (Suppl 3): S26-S32, 2011.

9. Vezzani A, Ravizza T, Balosso S and Aronica E: Glia as a source of cytokines: Implications for neuronal excitability and survival. Epilepsia 49 (Suppl 2): S24-S32, 2008.

10. Bizière K and Chambon JP: Animal models of epilepsy and experimental seizures. Rev Neurol (Paris) 143: 329-340, 1987 (In French).

11. Fisher RS: Animal models of the epilepsies. Brain Res Brain Res Rev 14: 245-278, 1989.

12. Twyman RE, Green RM and MacDonald RL: Kinetics of open channel block by penicillin of single GABAA receptor channels from mouse spinal cord neurons in culture. J Physiol 445: 97-127, 1992.

13. Qiu YY, Zhu JX, Bian T, Gao F, Qian XF, Du Q, Yuan MY, Sun h, Shi LZ and Yu H: Protective effects of Astragaloside IV agains ovalbumin-induced lung inflammation are regulated/mediated by T-bet/GATA-3. Pharmacol 94: 51-59, 2014.

14. Qiu L, Yin G, Cheng L, Fan Y, Xiao W, Yu G, Xing M, Jia R, Sun R, Ma X, et al: Astragaloside IV ameliorates acute pancreatitis in rats by inhibiting the activation of nuclear factor- $\kappa \mathrm{B}$. Int J Mol Med 35: 625-636, 2015.

15. Özdemir MB, Akça H, Erdoğan Ç, Tokgün O, Demiray A, Semin F and Becerir C: Protective effect of insulin and glucose at different concentrations on penicillin-induced astrocyte death on the primer astroglial cell line. Neural Regen Res 7: 1895-1899, 2012.
16. Livak KJ and Schmittgen TD: Analysis of relative gene expression data using real-time quantitative PCR and the 2(-Delta Delta C(T)) method. Methods 25: 402-408, 2001.

17. Hirtz D, Thurman DJ, Gwinn-Hardy K, Mohamed M, Chaudhuri AR and Zalutsk R: How common are the 'common' neurologic disorders?. Neurol 68: 326-337, 2007.

18. Brodie MJ, Elder AT and Kwan P: Epilepsy in later life. Lancet Neurol 8: 1019-1030, 2009.

19. Holmes, Thomas R. Browne, Gregory L: Handbook of epilepsy. 4th edition. Philadelphia, Lippincott Williams \& Wilkins pp7. ISBN 978-0-7817-7397-3, 2008.

20. Noebels JL, Avoli M, Rogawski MA, et al: Jasper's Basic Mechanisms of the Epilepsies. Oxford University Press, pp466-470, 2012.

21. Bromfield EB, Cavazos JE and Sirven JI: An introduction to epilepsy. American Epilepsy Society, 2006.

22. Blumenfeld $\mathrm{H}$ : Cellular and network mechanisms of spike-wave seizures. Epilepsia 46: 21-33, 2005.

23. Sofroniew MV and Vinters HV: Astrocytes: Biology and pathology. Acta Neuropathol 119: 7-35, 2010.

24. Vezzani A, Maroso M, Balosso S, Sanchez MA and Bartfai T: IL-1 receptor/Toll-like receptor signaling in infection, inflammation, stress and neurodegeneration couples hyperexcitability and seizures. Brain Behav Immun 25: 1281-1289, 2011.

25. Vezzani A, French J, Bartfai T and Baram TZ: The role of inflammation in epilepsy. Nat Rev Neurol 7: 31-40, 2011.

26. Vezzani A, Balosso S and Ravizza T: The role of cytokines in the pathophysiology of epilepsy. Brain Behav Immun 22: 797-803, 2008.

27. Balosso S, Ravizza T, Perego C, Peschon J, Campbell IL, De Simoni MG and Vezzani A: Tumor necrosis factor-alpha inhibits seizures in mice via p75 receptors. Ann Neurol 57: 804-812, 2005

28. Ravizza T, Lucas SM, Balosso S, Bernardino L, Ku G, Noé F, Malva J, Randle JC, Allan S and Vezzani A: Inactivation of caspase-1 in rodent brain: A novel anticonvulsive strategy. Epilepsia 47: 1160-1168, 2006.

29. Bellinger FP, Madamba S and Siggins GR: Interleukin 1 beta inhibits synaptic strength and long-term potentiation in the rat CA1 hippocampus. Brain Res 628: 227-234, 1993.

30. Schneider H, Pitossi F, Balschun D, Wagner A, del Rey A and Besedovsky H: A neuromodulatory role of interleukin-1beta in the hippocampus. Ann NY Acad Sci 95: 7778-7783, 1998.

31. Allan SM, Tyrrell PJ and Rothwell NJ: Interleukin-1 and neuronal injury. Nat Rev Immunol 5: 629-640, 2005. 